\title{
Helicobacter pylori is associated with weakened pulmonary function and reduced incidence of allergic conditions in patients with chronic cough
}

\author{
MENG HE ${ }^{1 *}$, YUANYUAN ZHENG ${ }^{2 *}$, A'HUO MA $^{2 *}$, CHUNYI ZHANG $^{1}$, YUEFANG YU ${ }^{1}$, \\ HUA WANG $^{1}$, YEFENG CHEN ${ }^{1}$, MIN XIANG $^{1}$, KELONG TAO $^{3}$ and JUXIN SHEN ${ }^{1}$ \\ Departments of ${ }^{1}$ Respiratory Medicine, ${ }^{2}$ Gastroenterology and ${ }^{3}$ Gastrointestinal Surgery, \\ Shaoxing People's Hospital (Shaoxing Hospital, Zhejiang University School of Medicine), Zhejiang University School of \\ Medicine, Shaoxing, Zhejiang 312000, P.R. China
}

Received July 26, 2019; Accepted June 10, 2020

DOI: $10.3892 /$ etm.2020.9176

\begin{abstract}
The present study aimed to investigate the potential association between Helicobacter pylori (a H.pylori) positive state and chronic cough. A clinical observational study with systematic analysis was performed, including 278 patients with complaints of chronic cough and 148 healthy controls. a $H$. pylori positive state was present in $61.2 \%$ of the patients in the chronic cough group and $68.9 \%$ in the chronic refractory cough group, as opposed to $43.9 \%$ in the control group. There was a significant improvement in $65.5 \%$ of the patients with chronic refractory cough following successful a $H$. pylori eradication therapy. In addition, patients with chronic cough exposed to a $H$. pylori exhibited decreased pulmonary function with a decrease in forced expiratory volume in $1 \mathrm{sec}$ by $84 \mathrm{ml}$, a decrease in the forced vital capacity by $53 \mathrm{ml}$ and a decrease in maximal vital capacity by $46 \mathrm{ml}$. The difference was even more obvious in the chronic refractory cough group. The allergy status differed significantly according to age between a $H$. pylori-positive and -negative cases in the cough variant asthma and allergic cough groups. Among
\end{abstract}

Correspondence to: Dr Kelong Tao, Department of Gastrointestinal Surgery, Shaoxing People's Hospital (Shaoxing Hospital, Zhejiang University School of Medicine), Zhejiang University School of Medicine, 568 Zhongxing North Road, Shaoxing, Zhejiang 312000, P.R. China

E-mail: fykelong@163.com

Professor Juxin Shen, Department of Respiratory Medicine, Shaoxing People's Hospital (Shaoxing Hospital, Zhejiang University School of Medicine), Zhejiang University School of Medicine, 568 Zhongxing North Road, Shaoxing, Zhejiang 312000, P.R. China E-mail: sxrmsjx@126.com

${ }^{*}$ Contributed equally

Key words: Helicobacter pylori, chronic cough, chronic refractory cough, cough variant asthma, allergic cough, lung function, atopy, immunoglobulin E, hypersensitivity patients aged $<40$ years, a $H$. pylori-positive cases had a lower prevalence of atopy and lower total serum immunoglobin $\mathrm{E}$ levels compared with a $H$. pylori-negative cases. However, there was no significant association between a $H$. pylori status and C-reactive protein levels, erythrocyte sedimentation rate or eosinophil count in the peripheral blood. In conclusion, the present study demonstrated that a $H$. pylori infection may be a factor associated with chronic cough and it may be associated with a decline in pulmonary function and reduced incidence of allergic conditions. Thus, a H.pylori may represent a target for the treatment of chronic cough.

\section{Introduction}

Chronic cough (CC) is a common clinical condition that may disrupt the everyday activities of affected patients, including their work. The prevalence of CC among the adult population has been reported to range between 13 and 32\% (1). By definition, $\mathrm{CC}$ is a cough lasting for $>8$ weeks in patients who have a normal chest radiograph, are not receiving therapy with angiotensin-converting enzyme inhibitors and have not been exposed to environmental irritants $(1,2)$. According to the data reported in the literature, cough variant asthma (CVA), upper airway cough syndrome (UACS), allergic cough (AC), eosinophilic bronchitis (EB) and gastroesophageal reflux-related cough (GERC) are the major causes of CC, accounting for $\sim 70-95 \%$ of the causes in Chinese patients $(3,4)$. Certain patients may have more than one of these conditions. However, in a significant number of patients, the cause of the cough may remain elusive, or the cough may be refractory to treatment, despite extensive investigations and therapeutic trials. Unexplained chronic cough (UCC) is a major health concern that occurs in up to $5-10 \%$ of the Chinese population $(5,6)$. Furthermore, although the aetiology may be identified in certain patients, there is no effective treatment method or there may be no treatment response, leading to persistent cough (7). Collectively, UCC and persistent cough with poor therapeutic response are referred to as chronic refractory cough (CRC), which occurs in up to $12-42 \%$ of cases (8). 
Chronic persistent cough may interrupt patients' sleep, leading to exhaustion. Affected patients experience a marked decline in their quality of life. They frequently seek medical help at various hospitals throughout China, are repeatedly subjected to a variety of examinations and receive a number of different drugs, suffer from various adverse drug reactions and carry a heavy financial burden (9). Furthermore, persistent respiratory symptoms, particularly in young adults, are associated with an accelerated decline in lung function, an increased incidence of obstructive and restrictive physiological conditions and an elevated risk of future lung diseases (10).

Helicobacter pylori (a H. pylori) is a slow-growing, microaerophilic, gram-negative bacterium; its most striking biochemical characteristic is the abundant production of urease. The prevalence of a $H$. pylori positive state varies from $<40 \%$ in developed countries to $>70 \%$ in developing countries (11). a $H$. pylori positive state is causally associated with various health conditions, including chronic active gastritis, peptic ulcer disease and gastric cancer (12). Over the past few years, a variety of extradigestive disorders, particularly respiratory system diseases, including chronic bronchitis, bronchiectasis, pulmonary tuberculosis, asthma, chronic obstructive pulmonary disease (COPD) and idiopathic pulmonary fibrosis (IPF), have been reported to be associated with a $H$. pylori positive state $(13,14)$. In addition, a $H$. pylori has been detected in the tracheobronchial aspirates of mechanically ventilated patients and the possibility that it may cause ventilator-associated pneumonia has been raised. Furthermore, previous studies have investigated a potential role for a $H$. pylori positive state in several clinical conditions (15). Their results demonstrated that a $H$. pylori positive state may be associated with several clinical manifestations (16-18), including chronic persistent cough. However, the exact underlying mechanisms have remained elusive. The current retrospective study was performed to investigate the role of a $H$. pylori positive state in $\mathrm{CC}$.

\section{Materials and methods}

Patients and selection criteria. A total of 278 adult patients referred to the respiratory clinic of Shaoxing People's Hospital (Shaoxing, China) due to chronic persistent cough over a 2-year enrolment period (June 2016-May 2018), including outpatients and inpatients, were enrolled in the present study. The control group was recruited from the Physical Examination Centre of Shaoxing People's Hospital (Shaoxing, China) and included 148 age- and sex-matched subjects with no history of CC, of whom 87 were females and 61 were males (female/male ratio, 1.4:1), with a mean age of 48.6 years (95\% CI: 46.53-50.75). The CC group included 176 females and 102 males (female/male ratio, 1.7:1), with a mean age of 48.2 years (95\% CI: 34.86-61.46).

The patients with different types of cough were required to meet the following inclusion and exclusion criteria: i) The major or only clinical symptom is a cough lasting for $>8$ weeks. ii) No exposure to environmental irritants. iii) Normal or near-normal chest radiographs or chest CT scans (no or minor stable inconsequential scarring). iv) No treatment with angiotensin-converting enzyme inhibitors. v) No response to antibiotic therapy. vi) Exclusion of chronic bronchitis, bronchiectasis, typical asthma, COPD and interstitial lung disease. vii) Exclusion of combination of two or more causes of CC.

Diagnosis. The diagnostic criteria according to Guidelines for diagnosis and treatment of cough (version 2015) (19) for the different types of cough are described below.

$C R C$. Persistent CC despite thorough investigation and conventional treatment according to published practice guidelines.

CVA. i) CC, usually irritating cough at night. ii) A positive result through bronchial provocation tests or bronchial dilation test or lung function mean diurnal peak expiratory flow (PEF) variation rate index $>10 \%$ (continuous detection for at least 7 days). iii) Cough improved significantly or even disappeared following anti-asthmatic treatments.

$A C$. i) CC, usually irritating dry cough. ii) Normal pulmonary ventilation and a negative result through bronchial provocation tests. iii) Normal numbers of sputum eosinophils. iv) A positive result in detection of atopy or total serum immunoglobulin (Ig) E levels or a history of allergic diseases or exposure to allergenic substances. v) Cough improved significantly or even disappeared after glucocorticoid or anti-histamine treatments.

$U A C S$. i) Paroxysmal or persistent cough occurring more frequently in the daytime, with disappearance or decrease during sleep. ii) Clinical manifestation and medical history of nasal or pharyngeal diseases. iii) Adjunctive examination supporting the diagnosis of nasal or pharyngeal diseases. iv) Cough improved significantly or even disappeared after treatment of the nasal or pharyngeal diseases.

GERC. i) CC occurring mainly in the daytime, associated with typical reflux symptoms (e.g. chest pain) or food intake. ii) Symptom index $\geq 45 \%$ or DeMeester score $\geq 12.7$ (24 h ambulatory oesophageal $\mathrm{pH}$ monitoring) or symptom association probability $\geq 80 \%$. iii) Cough improved significantly or even disappeared after anti-reflux therapy.

$E B$. i) CC, usually irritating dry cough, or cough with a small amount of phlegm. ii) Normal pulmonary ventilation, no airway hyperresponsiveness and normal lung function mean diurnal PEF variation rate index. iii) Percentage of sputum eosinophils $\geq 2.5 \%$. iv) Exclusion of other diseases characterised by eosinophil infiltration and proliferation. v) Cough improved significantly or even disappeared after oral or inhaled glucocorticoid treatment.

Other rare causes. i) Psychogenic cough: a) CC occurring only in the daytime with disappearance during sleep or while focusing on something. b) CC aggravated by a variety of psychological factors. c) A negative result following thorough investigation of CC. d) Exclusion of other causes of CC. e) Cough improved significantly or even disappeared after psychotherapy, anti-anxiety or anti-depressant therapy. ii) Post-infectious cough: a) CC persisting after the disappearance of acute symptoms of respiratory infections, usually irritating dry cough or with a small amount of phlegm. 
Table I. Patient characteristics.

\begin{tabular}{lccc}
\hline Characteristics & CC group $(\mathrm{n}=278)$ & Control group $(\mathrm{n}=148)$ & P-value \\
\hline Sex, female/male & $176 / 102$ & $87 / 61$ & 0.36 \\
Age, years & $48.16 \pm 13.30$ & $48.64 \pm 13.00$ & 0.289 \\
Body height, cm & $162.91 \pm 7.63$ & $162.85 \pm 7.27$ & 0.942 \\
Body weight, kg & $62.38 \pm 9.92$ & $59.88 \pm 11.00$ & 0.018 \\
BMI, kg/m & $23.44 \pm 2.86$ & $22.48 \pm 3.19$ & 0.22 \\
Education, years & $9.80 \pm 4.20$ & $10.21 \pm 3.72$ & 0.517 \\
Smoking & & & \\
Pack years & $2.73 \pm 8.12$ & $4.43 \pm 11.06$ & $0.21 \pm 1.24$ \\
Time since & $0.24 \pm 1.60$ & & 0.089 \\
smoking cessation, years & & &
\end{tabular}

Continuous variables are expressed as the mean \pm standard deviation. BMI, body mass index.

b) Normal or near-normal (no more than stable inconsequential scarring) chest radiographs or chest CT scans; c) Exclusion of other causes of $\mathrm{CC}$; d) Cough improved significantly or even disappeared after anti-tussive, anti-histamine or decongestant treatment.

Evaluation of cough severity. A self-administered symptom telephone questionnaire was used to evaluate cough severity and its effect on the quality of life on a ranking scale, with scores of 1,2, 3 and 4 indicating mild, moderate, severe and highly severe complaints with high impairment of life quality, respectively.

Occasional cough was graded as mild (cough symptom score, 1).

Intermittent cough without affecting the quality of life (including the quality of sleep) was graded as moderate (cough symptom score, 2).

Intermittent cough with a mild impairment of life quality (including the quality of sleep) was graded as severe (cough symptom score, 3).

Persistent cough with a serious impairment of life quality (including the quality of sleep) was graded as highly severe (cough symptom score, 4).

Clinical examination. The medical history of patients with $\mathrm{CC}$ frequently provided important initial clues. The history of the patients included in the present study was thoroughly recorded, including meticulous assessment of the symptoms, by using telephone interviews. The concurrent symptoms may suggest a suitable diagnosis. At the Shaoxing People's Hospital, $\mathrm{CC}$ is diagnosed strictly according to the diagnostic criteria outlined above. Subsequently, the necessary examinations and experimental treatments may be performed to make an accurate diagnosis and clearly determine the causes of the CC.

Clinical examinations were performed, including chest $\mathrm{X}$-ray or $\mathrm{CT}$, lung function measurements, including the bronchodilatation test or bronchial provocation test, esophagogastroduodenoscopy or 24-h ambulatory oesophageal $\mathrm{pH}$ monitoring, nasal endoscopy, laryngoscopy, paranasal sinus $\mathrm{X}$-ray or CT, induced sputum eosinophils, bronchoscopy and detection of atopy, total serum IgE levels, C-reactive protein (CRP), erythrocyte sedimentation rate (ESR) and eosinophil (EO) count in the peripheral blood. IgE levels $>200 \mathrm{IU} / \mathrm{ml}$ were considered positive. A a $H$. pylori positive state was assayed using the ${ }^{13} \mathrm{C}$ or ${ }^{14} \mathrm{C}$ urea exhalation test (01 tester; Shenzhen Zhonghe Headway Biological Technology Co. Ltd.). All the participants were examined on an empty stomach in the morning.

Certain patients with a $H$. pylori positive state received a bismuth-containing quadruple therapy consisting of amoxicillin (1 g twice daily), clarithromycin (500 $\mathrm{mg}$ twice daily) and omeprazole (20 mg twice daily) for 14 days (20). The a $H$. pylori status was followed up for 6 weeks after the completion of treatments. a H.pylori eradication treatment was offered based on the gastroenterologists' discretion according to the American College of Gastroenterology Clinical Guidelines (version 2017) (21).

Statistical analysis. SPSS (version 20.0; IBM Corp.) statistical software was used for data analysis. Multivariate analysis was used to investigate the independent association of a $H$. pylori and CC. Comparisons of log-transformed CRP and ESR values, blood EO count, lung function indices [forced expiratory volume in $1 \mathrm{sec}$ (FEV1), forced vital capacity (FVC), maximal vital capacity (VCmax)] as well as body height, body weight and body mass index (BMI) between groups were performed by an unpaired Student's t-test. The $\chi^{2}$ test was used to compare the prevalence of a $H$. pylori positive state, symptom improvement rate, atopy, serum IgE levels and other data including sex, age, educational background and smoking status among groups. $\mathrm{P}<0.05$ was considered to indicate a statistically significant difference.

\section{Results}

Patient characteristics. The present study included a total of 426 subjects, which were divided into two groups. The study group included 278 patients with chronic persistent cough, who were then subdivided into 7 subgroups according to the causes of $\mathrm{CC}$ as described above (CC: Cough lasting for $>8$ weeks 
Table II. CC patient characteristics.

\begin{tabular}{lccc}
\hline Variable & H.pylori positive & H.pylori negative & P-value \\
\hline Number & 170 & 108 & 0.15 \\
Sex (male/female) & $68 / 102$ & $34 / 74$ & 0.07 \\
Age & $46.54 \pm 12.42$ & $50.71 \pm 14.27$ & 0.14 \\
Heigh (cm) & $163.45 \pm 7.54$ & $162.06 \pm 7.71$ & 0.74 \\
Weight (Kg) & $62.53 \pm 10.60$ & $62.13 \pm 8.80$ & 0.35 \\
BMI & $23.31 \pm 2.95$ & $23.64 \pm 2.73$ & 0.06 \\
Education (Years) & $9.49 \pm 4.38$ & $10.27 \pm 3.83$ & 0.25 \\
Smoking status Pack years & $2.87 \pm 8.03$ & $2.51 \pm 8.30$ & \\
\hline
\end{tabular}

Continuous data are shown as mean \pm standard deviation.

Table III. Control group patient characteristics.

\begin{tabular}{lccc}
\hline Variable & H. pylori positive & H.pylori negative & P-value \\
\hline Number & 65 & 83 & $31 / 52$ \\
Sex (male/female) & $30 / 35$ & $48.80 \pm 12.54$ & 0.28 \\
Age & $48.45 \pm 13.67$ & $162.69 \pm 7.23$ & 0.84 \\
Heigh (cm) & $163.06 \pm 7.36$ & $60.81 \pm 11.71$ & 0.76 \\
Weight (Kg) & $58.69 \pm 9.98$ & $22.86 \pm 3.35$ & 0.25 \\
BMI & $22.01 \pm 2.93$ & $11.11 \pm 3.36$ & 0.11 \\
Education(years) & $9.12 \pm 3.75$ & $3.50 \pm 10.20$ & 0.02 \\
Smoking status Pack years & $5.61 \pm 12.05$ & 0.06 \\
\hline
\end{tabular}

Continuous data are shown as mean \pm standard deviation.

in patients who exhibited a normal chest radiograph, are not receiving therapy with angiotensin-converting enzyme inhibitors and have not been exposed to environmental irritants such as occupational dust and chemical substances).

The control group recruited from the Physical Examination Centre of Shaoxing People's Hospital included 148 age- and sex-matched subjects with no history of CC or other respiratory diseases. No significant difference in sex, age, body height, body weight, BMI, educational background or smoking status was present between the $\mathrm{CC}$ and control groups $(\mathrm{P}>0.05)$. In the $\mathrm{CC}$ group, there was also no significant difference in sex, age, height, weight, BMI, educational background and smoking status between a $H$. pylori positive and negative cases $(\mathrm{P}>0.05)$. However, patients with a $H$. pylori positive (Tables I, II and III).

H. pylori positivity. The rate of $H$. pylori positivity was $170 / 278(61.2 \%)$ in the CC group and 65/148 (43.9\%) in the control group. The difference was indicated to be statistically significant $(\mathrm{P}<0.05)$. Furthermore, there were 42/61 (68.9\%) a $H$. pylori-positive cases in the CRC group $(\mathrm{P}<0.05), 69 / 113$ $(61.1 \%)$ in the CVA group $(\mathrm{P}<0.05), 16 / 26(61.5 \%)$ in the AC group $(\mathrm{P}>0.05), 13 / 20(65 \%)$ in the UACS group $(\mathrm{P}>0.05)$ and $22 / 37(59.5 \%)$ in the GERC group ( $P>0.05)$ compared with the control group. The remaining two groups were not
Table IV. Prevalence of $H$. pylori positivity.

\begin{tabular}{lrrr}
\hline Group & $\begin{array}{c}\text { H.pylori }(+), \\
\text { n/totals }(\%)\end{array}$ & $\begin{array}{c}\text { H.pylori }(-), \\
\text { n/totals }(\%)\end{array}$ & P-value \\
\hline CC & $170 / 278(61.2)$ & $108 / 278(38.8)$ & 0.001 \\
CRC & $42 / 61(68.9)$ & $19 / 61(31.1)$ & 0.001 \\
CVA & $69 / 113(61.1)$ & $44 / 113(38.9)$ & 0.006 \\
AC & $16 / 26(61.5)$ & $10 / 26(38.5)$ & 0.097 \\
UACS & $13 / 20(65.0)$ & $7 / 20(35.0)$ & 0.076 \\
GERC & $22 / 37(59.5)$ & $15 / 37(40.5)$ & 0.09 \\
EB & $5 / 11(45.5)$ & $6 / 11(54.5)$ & 0.921 \\
Other rare reasons & $5 / 10(50.0)$ & $5 / 10(50.0)$ & 0.708 \\
Control & $65 / 148(43.9)$ & $83 / 148(56.1)$ & \\
& & &
\end{tabular}

CC, chronic cough group; CRC, chronic refractory cough group; CVA, cough variant asthma group; AC, allergic cough group; UACS, upper airway cough syndrome group; GERC, gastroesophageal reflux-associated cough group; EB, eosinophilic bronchitis.

analysed due to insufficient numbers of patients. The results are presented in Table IV. 
Table V. Pulmonary function.

\begin{tabular}{|c|c|c|c|c|c|c|}
\hline \multirow[b]{2}{*}{ Group } & \multicolumn{2}{|c|}{ FEV1, 1} & \multicolumn{2}{|c|}{$\mathrm{FVC}, 1$} & \multicolumn{2}{|c|}{ VCmax, 1} \\
\hline & H.pylori $(+)$ & H.pylori (-) & H.pylori (+) & H.pylori (-) & H.pylori (+) & H.pylori (-) \\
\hline $\mathrm{CC}$ & $2.85 \pm 0.74$ & $2.93 \pm 0.84$ & $3.59 \pm 0.89$ & $3.65 \pm 0.99$ & $3.70 \pm 0.91$ & $3.75 \pm 0.99$ \\
\hline $\mathrm{CRC}$ & $2.98 \pm 0.61^{\mathrm{a}}$ & $3.37 \pm 0.85$ & $3.74 \pm 0.69$ & $4.08 \pm 1.04$ & $3.86 \pm 0.72$ & $4.17 \pm 1.01$ \\
\hline CVA & $2.62 \pm 0.71$ & $2.80 \pm 0.90$ & $3.37 \pm 0.85$ & $3.56 \pm 1.04$ & $3.46 \pm 0.86$ & $3.68 \pm 1.06$ \\
\hline $\mathrm{AC}$ & $2.92 \pm 0.58$ & $2.82 \pm 0.86$ & $3.59 \pm 0.89$ & $3.40 \pm 1.09$ & $3.74 \pm 0.86$ & $3.51 \pm 1.08$ \\
\hline UACS & $2.91 \pm 0.91$ & $2.89 \pm 0.71$ & $3.69 \pm 1.12$ & $3.59 \pm 0.90$ & $3.76 \pm 1.18$ & $3.61 \pm 0.88$ \\
\hline GERC & $3.25 \pm 0.86$ & $2.97 \pm 0.75$ & $4.04 \pm 1.03$ & $3.66 \pm 0.96$ & $4.20 \pm 1.03$ & $3.73 \pm 0.99$ \\
\hline Control & $2.94 \pm 0.69$ & $2.92 \pm 0.78$ & $3.63 \pm 0.85$ & $3.63 \pm 0.95$ & $3.75 \pm 0.87$ & $3.71 \pm 0.96$ \\
\hline
\end{tabular}

Values are expressed as the mean \pm standard deviation. ${ }^{\mathrm{a}} \mathrm{P}=0.044$ vs. $H$. pylori (-). CC, chronic cough group; CRC, chronic refractory cough group; CVA, cough variant asthma group; AC, allergic cough group; UACS, upper airway cough syndrome group; GERC, gastroesophageal reflux-associated cough group; EB, eosinophilic bronchitis; FEV1, forced expiratory volume in 1 sec; FVC, forced vital capacity; VCmax, maximal vital capacity.

Table VI. Predicted pulmonary function $(\%)$.

\begin{tabular}{|c|c|c|c|c|c|c|}
\hline \multirow[b]{2}{*}{ Group } & \multicolumn{2}{|c|}{ FEV1 } & \multicolumn{2}{|c|}{ FVC } & \multicolumn{2}{|c|}{ VCmax } \\
\hline & H.pylori (+) & H.pylori (-) & H.pylori (+) & H. pylori (-) & H.pylori $(+)$ & H. pylori (-) \\
\hline $\mathrm{CC}$ & $100.79 \pm 14.80^{\mathrm{a}}$ & $107.32 \pm 15.56$ & $104.78 \pm 14.85^{\mathrm{a}}$ & $109.00 \pm 15.48$ & $105.41 \pm 14.30^{\mathrm{a}}$ & $109.00 \pm 14.50$ \\
\hline $\mathrm{CRC}$ & $102.24 \pm 8.33^{\mathrm{a}}$ & $115.23 \pm 10.82$ & $106.06 \pm 10.65^{a}$ & $115.82 \pm 10.81$ & $106.51 \pm 10.45^{\mathrm{a}}$ & $115.62 \pm 10.87$ \\
\hline CVA & $96.15 \pm 15.02$ & $101.05 \pm 16.26$ & $101.85 \pm 14.39$ & $104.52 \pm 15.96$ & $102.37 \pm 14.05$ & $104.61 \pm 14.40$ \\
\hline $\mathrm{AC}$ & $106.11 \pm 18.73$ & $108.41 \pm 15.62$ & $106.00 \pm 21.00$ & $106.50 \pm 13.66$ & $107.32 \pm 18.93$ & $107.42 \pm 14.05$ \\
\hline UACS & $98.01 \pm 13.10^{\mathrm{a}}$ & $112.19 \pm 11.32$ & $103.68 \pm 16.93$ & $114.30 \pm 15.83$ & $102.48 \pm 17.18$ & $112.06 \pm 14.48$ \\
\hline GERC & $110.58 \pm 15.32$ & $109.12 \pm 13.68$ & $111.94 \pm 15.40$ & $110.15 \pm 17.97$ & $113.74 \pm 13.74$ & $109.61 \pm 17.76$ \\
\hline Control & $105.80 \pm 12.10$ & $107.58 \pm 16.28$ & $107.18 \pm 15.36$ & $109.46 \pm 16.09$ & $107.69 \pm 13.88$ & $108.78 \pm 15.76$ \\
\hline
\end{tabular}

${ }^{a} \mathrm{P}<0.05$. Values are expressed as the mean \pm standard deviation. $\mathrm{CC}$, chronic cough group; $\mathrm{CRC}$, chronic refractory cough group; $\mathrm{CVA}$, cough variant asthma group; AC, allergic cough group; UACS, upper airway cough syndrome group; GERC, gastroesophageal reflux-associated cough group; EB, eosinophilic bronchitis; FEV1, forced expiratory volume in 1 sec; FVC, forced vital capacity; VCmax, maximal vital capacity.

Pulmonary function. Patients with CC exposed to a $H$. pylori exhibited lower lung function as compared with those who were not, with a decrease in FEV1 by $84 \mathrm{ml}$, a decrease in FVC by $53 \mathrm{ml}$ and a decrease in VCmax by $46 \mathrm{ml}(\mathrm{P}>0.05)$. Although the difference did not reach statistical significance, it became statistically significant after adjusting for height, weight, age, sex and ethnicity (the adjusted value was expressed as percent predicted pulmonary function, $\mathrm{P}<0.05)$. There was no significant difference in the aforementioned pulmonary function indices between a $H$. pylori-positive and -negative cases in the control group ( $\mathrm{P}>0.05$; Table VI).

Furthermore, a subgroup analysis was performed. Patients in the CRC group exposed to a H.pylori exhibited lower lung function compared with those who were not, with a decrease in FEV1 by $395 \mathrm{ml}(\mathrm{P}<0.05)$, a decrease in FVC by $335 \mathrm{ml}$ $(\mathrm{P}>0.05)$ and a decrease in VCmax by $314 \mathrm{ml}(\mathrm{P}>0.05)$. After adjusting for height, weight, age, sex and ethnicity, effect modification was observed for the association between a $H$. pylori positive state and pulmonary function in the CRC group $(\mathrm{P}<0.05)$. The percent predicted pulmonary function was calculated using a prediction formula according to epidemiological survey data of the East China area from Shanghai Zhongshan Hospital, Fudan University (22). The results are presented in Tables V and VI and Figs. 1 and 2.

Atopy and total IgE. Patients with CC who were a $H$. pylori-positive had fewer allergic conditions compared with those who were a $H$. pylori-negative, as evaluated by determination of atopy and total serum IgE levels $(\mathrm{P}<0.05)$. There was no statistically significant association between a $H$. pylori exposure and allergic conditions in the CVA and AC groups. However, the subgroup analysis according to age revealed an inverse association between a $H$. pylori positive state and allergic conditions in those patients. The allergic status differed significantly according to age between a $H$. pylori-positive and -negative cases in the CVA and AC groups. Among the patients aged $<40$ years, a $H$. pylori-positive patients had a lower prevalence of atopy compared with a $H$.pylori-negative 


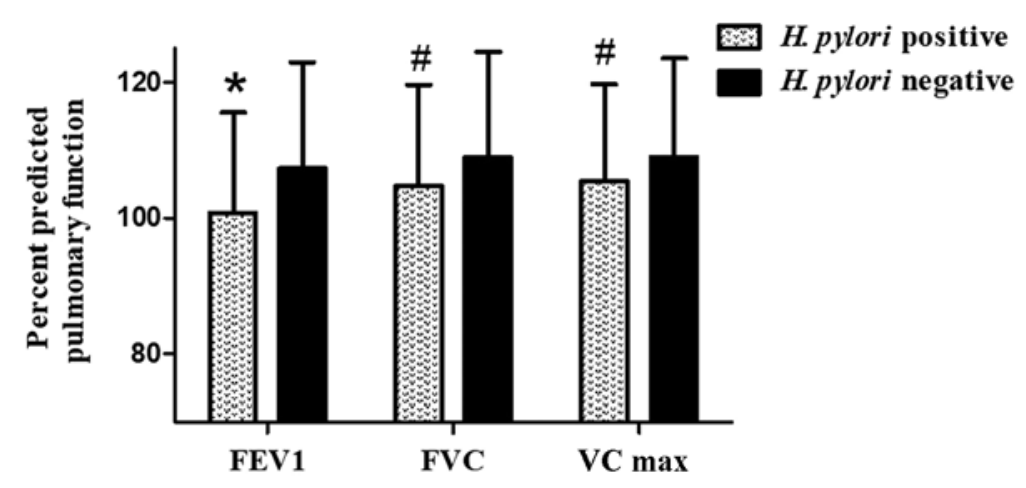

Figure 1. Predicted pulmonary function in the CC group. CC patients with a H.pylori positive had lower lung function than those who were not after adjusting for factors including height, weight, age, sex and ethnicity. The adjusted value was expressed as percent predicted pulmonary function. Values: "P<0.001 and ${ }^{\text {"}} \mathrm{P}<0.05$ vs. negative group. FEV1, forced expiratory volume in $1 \mathrm{sec} ; \mathrm{FVC}$, forced vital capacity; VCmax, maximal vital capacity.

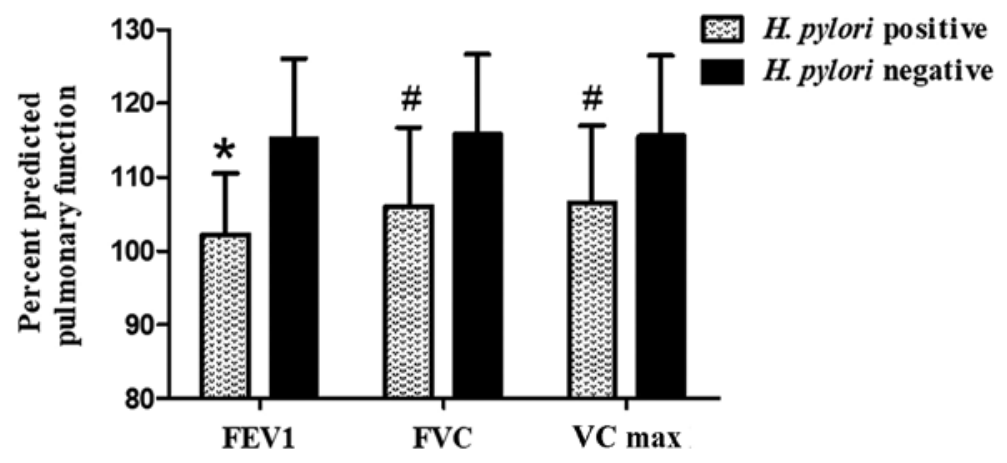

Figure 2. Predicted pulmonary function in the chronic refractory cough group. Patients in the chronic refractory cough group exposed to a $H$. pylori had lower lung function than those who were not after adjusting for factors including height, weight, age, sex and ethnicity. The adjusted value was expressed as percent predicted pulmonary function. Values: A, 102.24 $\pm 8.3(\mathrm{P}<0.001) ; \mathrm{B}, 115.23 \pm 10.8 ; \mathrm{C}, 106.06 \pm 10.6(\mathrm{P}=0.002) ; \mathrm{D}, 115.82 \pm 10.8 ; \mathrm{E}, 106.51 \pm 10.4(\mathrm{P}=0.003)$; $\mathrm{F}, 115.62 \pm 10.9$. ${ }^{*} \mathrm{P}<0.001$ and ${ }^{\#} \mathrm{P}<0.05$ vs. negative group. FEV1, forced expiratory volume in 1 sec; FVC, forced vital capacity; VCmax, maximal vital capacity.

cases (46.9 vs. $81.8 \%, \mathrm{P}<0.05)$. After defining serum IgE levels $>200 \mathrm{IU} / \mathrm{ml}$ as positives, there was a significantly reduced number of IgE-positive patients in the $H$. pylori-positive subgroup among patients aged $<40$ years $(37.5$ vs. $72.7 \%$, $\mathrm{P}<0.05)$. The results are provided in Table VII.

$C R P, E S R$ and EO in the peripheral blood. There was no significant association between $H$. pylori status and CRP, ESR or EO count in the peripheral blood following the $\log$-transformation $(\mathrm{P}>0.05)$. The results of the subgroup analysis according to the cause of $\mathrm{CC}$ were consistent $(\mathrm{P}>0.05$; Data not shown). The results are presented in Table VIII.

Effect of a H. pylori eradication. Patients with CC exposed to H. pylori had higher cough symptom scores and poorer therapeutic responses (cough symptom score: $2.92 \pm 0.93$ in $H$. pylori-positives vs. $2.50 \pm 0.92$ in $H$. pylori-negatives; $\mathrm{P}<0.001)$. The patients with CRC were followed up. A total of 29 patients with CRC received $H$. pylori eradication treatment, 19 of whom exhibited an improvement in the cough and the cough-specific quality of life at 1-2 months after successful eradication (improvement was defined as a reduction in the ranking scores of the evaluated symptoms of at least 1 ). However, a long-term follow-up study is required to fully elucidate the effect of $H$. pylori eradication on pulmonary function. The results are presented in Table IX.

\section{Discussion}

Since Warren and Marshall (23) reported on the culture of a spiral bacterium from the stomach in 1983, subsequently named a $H$. pylori, the causative role of this gram-negative bacterium in various upper-gastrointestinal-tract diseases has been increasingly recognized $(24,25)$. With advancing research, several studies focused on the association between a H. pylori positive state and extra digestive diseases, including respiratory system diseases $(26,27)$. It was reported by scholars from Alexandria University that a H. pylori positive state may lead to several clinical manifestations of respiratory-tract diseases (15). Furthermore, a H. pylori has been identified in the tracheobronchial aspirates of mechanically ventilated patients and may cause ventilator-associated pneumonia (28). This prompted us to investigate the possible association between a $H$. pylori positive state and CC, particularly CRC.

The present study revealed that a high percentage (up to $61.2 \%$ ) of patients with CC suffered from active a $H$. pylori infection (29), particularly those with CRC (up to 68.9\%). This was significantly higher compared with the percentage of active a $H$. pylori positive state among controls without cough (43.9\%). Subsequently, a follow-up study was performed by telephone interviews. The results revealed that $65.5 \%(19 / 29)$ of patients with CRC who had received a $H$.pylori eradication treatment exhibited an improvement in their cough and the 
Table VII. Association between $H$. pylori exposure and allergic conditions.

\begin{tabular}{|c|c|c|c|c|}
\hline Groups & Atopy (+), n/total (\%) & P-value & IgE, n/total (\%) & P-value \\
\hline \multicolumn{5}{|l|}{ Chronic cough } \\
\hline H.pylori $(+)$ & $46 / 170(27.1)$ & $<0.05$ & $46 / 170(27.1)$ & $<0.05$ \\
\hline HP (-) & 43/108 (39.8) & & 44/108 (40.7) & \\
\hline \multicolumn{5}{|l|}{$\mathrm{CVA}$ and $\mathrm{AC}$} \\
\hline H.pylori $(+)$ & $60 / 88(68.2)$ & $>0.05$ & $44 / 88(50.0)$ & $>0.05$ \\
\hline H.pylori (-) & $35 / 53(66.0)$ & & $33 / 53(62.3)$ & \\
\hline \multicolumn{5}{|c|}{ Age $<40$ years $(\mathrm{CVA}$ and $\mathrm{AC})$} \\
\hline H.pylori $(+)$ & $15 / 32(46.9)$ & $<0.05$ & $12 / 32(37.5)$ & $<0.05$ \\
\hline H.pylori (-) & 9/11 (81.8) & & $8 / 11(72.7)$ & \\
\hline \multicolumn{5}{|l|}{ Control group } \\
\hline H.pylori $(+)$ & $9 / 65(13.8)$ & $>0.05$ & $4 / 65(6.2)$ & $>0.05$ \\
\hline H.pylori $(-)$ & $9 / 83(10.8)$ & & $6 / 83(7.2)$ & \\
\hline
\end{tabular}

CVA, cough variant asthma group; AC, allergic cough group.

Table VIII. Association between H. pylori exposure and systemic inflammation.

\begin{tabular}{lccc}
\hline Group & $\begin{array}{c}\text { H.pylori }(+), \\
\mathrm{n}(\%)\end{array}$ & $\begin{array}{c}\text { H.pylori }(-), \\
\mathrm{n}(\%)\end{array}$ & P-value \\
\hline Chronic cough & & & \\
CRP & $2.24 \pm 6.54$ & $3.43 \pm 10.68$ & 0.309 \\
Log CRP & $-0.01 \pm 0.46$ & $0.03 \pm 0.51$ & 0.511 \\
ESR & $10.35 \pm 8.31$ & $11.78 \pm 13.42$ & 0.419 \\
Log ESR & $0.88 \pm 0.36$ & $0.88 \pm 0.40$ & 0.943 \\
EO & $0.16 \pm 0.18$ & $0.16 \pm 0.32$ & 0.914 \\
Log EO & $-0.94 \pm 0.36$ & $-1.01 \pm 0.40$ & 0.175 \\
Control group & & & \\
CRP & $4.58 \pm 11.19$ & $3.02 \pm 6.23$ & 0.316 \\
Log CRP & $0.13 \pm 0.66$ & $0.05 \pm 0.60$ & 0.486 \\
ESR & $13.68 \pm 24.04$ & $13.91 \pm 18.10$ & 0.962 \\
Log ESR & $0.88 \pm 0.30$ & $0.88 \pm 0.35$ & 0.996 \\
EO & $0.13 \pm 0.11$ & $0.11 \pm 0.10$ & 0.326 \\
Log EO & $-1.02 \pm 0.38$ & $-1.10 \pm 0.41$ & 0.263 \\
\hline
\end{tabular}

Values are expressed as the mean \pm standard deviation. CRP, C-reactive protein; ESR, erythrocyte sedimentation rate; EO, eosinophil count in peripheral blood.

cough-associated quality of life at 1-2 months after successful eradication. Thus, it may be inferred from the results that there may be a potential association between a $H$.pylori positive state and CC. The results of the present study are consistent with those reported by a study from the Alexandria University (15).

While the pathogenetic mechanisms underlying the effects of a $H$. pylori on airway disorders remain to be determined, they may involve chronic inflammation promoted by persistent infection with a $H$. pylori $(30,31)$. It is well-known that a $H$. pylori colonization of the gastric mucosa stimulates the
Table IX. Cough symptom score in chronic cough and CRC groups (a $H$. pylori positive vs. a $H$.pylori negative).

\begin{tabular}{lccc}
\hline & $\begin{array}{c}\text { H.pylori } \\
\text { positive }\end{array}$ & $\begin{array}{c}\text { H.pylori } \\
\text { negative }\end{array}$ & P-value \\
\hline Cough symptom score & & & \\
Chronic cough & $2.92 \pm 0.93$ & $2.50 \pm 0.92$ & $\mathrm{P}<0.001$ \\
CRC & $3.19 \pm 0.86$ & $2.26 \pm 0.87$ & $\mathrm{P}<0.001$
\end{tabular}

Continuous data are shown as mean \pm standard deviation. Cough symptom score ranges from 1-4 (1: Mild; 2: Moderate; 3: Severe; 4: Extremely serious).

release of various pro-inflammatory factors, including cytokines and eicosanoids. The neutrophil-activating protein of a $H$. pylori is a major pro-inflammatory factor that not only has a key role in driving T-helper type 1 (Th1) inflammation, but is also able to inhibit Th2-mediated bronchial inflammation (32,33). Talaat et al (15) suggested that continuous release of inflammatory mediators by a $H$. pylori may result in direct or indirect effects on the oesophageal mucosa, which increased its sensitivity to acid and affected the motility of the oesophageal sphincter. As a result, exposure of the lower oesophagus to comparably short-term reflux episodes, through vagally mediated reflexes, may result in persistent cough.

Furthermore, a H.pylori is capable of adhering to epithelial cell lines originally derived from different organs, including respiratory tract epithelial cells. a $H$. pylori was recently identified in the tracheobronchial aspirates of mechanically ventilated patients and there may be an association between a $H$. pylori and ventilator-associated pneumonia (28). A study from Japan demonstrated that a $H$. pylori VacA, the major exotoxin of a H.pylori, was present in the human lung and induced inflammatory factor production by human lung cells (34). It is possible that a $H$. pylori is aspirated into the 
respiratory tract from the oropharynx or the gastric reservoir, thereby causing chronic persistent cough. This chronic microaspiration may be involved in chronic inflammatory injuries of the airway epithelia, as well as a systemic immune response that affects the respiratory system in susceptible patients. VacA may exert a local immunosuppressive effect. VacA inhibits the production of interleukin (IL)-2, which is required for T-cell viability and proliferation, and downregulates the surface expression of IL-2 receptor- $\alpha$ (32). In addition, it was reported that VacA induced apoptosis by release of cytochrome $\mathrm{c}$ from mitochondria and activation of caspase-3, which may lead to airway injury (35).

A clinical study from the Nottingham City Hospital demonstrated that individuals with a $H$. pylori positive state exhibited decreased lung function, with FEV1 being lower by $53 \mathrm{ml}$ and FVC by $83 \mathrm{ml}$ in their cross-sectional analysis, although this association was not independent of body height or the socioeconomic status (36). Similar results were reported by a Canadian study, which demonstrated that patients who were seropositive for a $H$. pylori had lower FEV1 values compared with individuals who were seronegative for a $H$. pylori; however, the significance of the association between FEV1 and a $H$. pylori seropositivity disappeared when FEV1 percent predicted values were used (37). Furthermore, previous studies demonstrated that a $H$. pylori positive state was associated with reduced lung function in patients with COPD and IPF, which is most likely due to the effect of the bacterium on lung growth earlier in life $(38,39)$. Systemic inflammation may also be a contributing factor.

The present study investigated the effect of a $H$. pylori positive state on lung function, as well as the systemic inflammation as evaluated by CRP, ESR, IgE and peripheral blood EO count in patients with $\mathrm{CC}$. The results demonstrated that patients exposed to a $H$. pylori in the $\mathrm{CC}$ group had a lower percent predicted lung function compared with those who were negative for $H$. pylori. The difference was even more obvious in the CRC group. Furthermore, there was no association between a $H$.pylori status and lung function in the control group. Therefore, it may be inferred that in patients with $\mathrm{CC}$, a $H$. pylori positive state is associated with decreased lung function, although further studies and a long-term follow-up are required to confirm this potential association. However, no significant association was observed between a $H$. pylori positive state and systemic inflammation markers, including CRP, ESR, IgE and EO count.

Persistent airway injury and inflammation cause airway remodelling and a progressive impairment of lung function, leading to chronic airway diseases. Studies have demonstrated that in young adults, persistent respiratory symptoms, including persistent cough, were associated with an accelerated decline in lung function, incidence of obstructive and restrictive physiology and a higher risk of future radiographic emphysema $(10,40)$. The potential association between a $H$. pylori positive state and $\mathrm{CC}$ indicates that the presence of cough-associated respiratory symptoms and persistent airway inflammation induced by a $H$.pylori may serve as a prognostic marker for accelerated decline in lung function and future lung diseases. This association was more obvious in patients with CRC.
A meta-analysis demonstrated that a $H$. pylori positive state was associated with an estimated $18 \%$ reduction in the risk of atopy, as estimated by allergen skin tests and specific $\operatorname{IgE}(41)$. Whether a $H$. pylori positive state is inversely associated with allergic diseases remains controversial (42-45). An experimental study demonstrated that oral immunomodulators with a H. pylori extract prevented airway hyperresponsiveness, bronchoalveolar eosinophilia, pulmonary inflammation and Th2 cytokine production, which are hallmarks of allergen-induced asthma, in mice (44). Similar results were also obtained in a recent study (45). Furthermore, several large cross-sectional and case-control studies demonstrated an inverse association between a $H$. pylori positive state and allergic diseases, including inflammatory airway diseases (44). However, others reported no association or a weak inverse association (41). In the present study, patients with $\mathrm{CC}$ who were a $H$. pyloripositive had fewer allergic conditions compared with those who were a $H$. pylori-negative, as evaluated by determination of atopy and total serum IgE levels. However, there was no statistically significant association between $H$. pylori exposure and allergic conditions in the CVA and AC groups. Of note, the subgroup analysis according to age revealed an inverse association between a $H$. pylori positive state and allergic conditions in those patients. Among those aged $<40$ years, a $H$. pylori-positive patients had a lower prevalence of atopy and serum IgE levels compared with a $H$. pylori-negative cases. The present results were in line with Korean studies, according to which the association of $\operatorname{IgE}$ hypersensitivity and allergic diseases with a $H$. pylori positive state differed depending on age $(44,46)$. Another study observed that early exposure to $H$. pylori was inversely associated with allergic conditions, which was possibly linked to infection with $\mathrm{CagA}^{+}$strains of H. pylori (41).

The gradual loss of the human indigenous microbiota may be implicated in the increasing trend in the prevalence of allergic diseases (42). H. pylori, as a bacterial pathogen, is a constituent of the normal gastric microbiota, which may partly explain the inverse association between a $H$. pylori positive state and allergic conditions in patients with CVA and AC aged $<40$ years (42). According to the 'decreasing microbiota hypothesis', the intestinal microbiota affects the immune system and commensal bacteria regulate the Th1/Th2 equilibrium. H. pylori, an ancient indigenous microbe, is expected to affect the immune system by shifting the cytokine balance toward the Th1 type, which suppresses Th2-dominated allergic diseases. It has been reported that $H$. pylori alters the T-cell response by inducing the expression of IL-12, tumour necrosis factor- $\alpha$ and interferon- $\gamma$ by T-cells. In the present study, an inverse association between $H$. pylori and allergic conditions in patients with CVA and AC aged $<40$ years, but not in those aged $>40$ years was identified. The reason for this remains elusive; however, it may be associated with the pathophysiological processes of CVA and AC, which also require further study. In addition, this is complicated by the fact that certain children may change back from a positive to a negative status of $H$. pylori positivity. In a population-based birth cohort in Ethiopia, $17 \%$ of children with evidence of a $H$.pylori positive state at the age of 3 years ceased to be negative at the age of 5 years (47). The issue of the fluctuating exposure status remains a major challenge. 
Patients with chronic persistent cough experience a significant reduction in their quality of life, particularly patients with refractory chronic persistent cough. Persistent cough in young adults was associated with accelerated decline in lung function, which was associated with a greater risk of future development of lung diseases, including COPD. Therefore, it is crucial to develop an effective treatment. The present study raised the possibility that $H$. pylori may be a cause of chronic cough, and that eradication of this pathogen may resemble a potential treatment. In certain patients with CRC, including those with unexplained chronic cough, the symptoms subsided after a successful $H$. pylori eradication, which may prevent the decline in lung function and airway remodelling. In addition, a vaccination for preventing or treating a $H$. pylori infection may be of value, particularly during childhood. Since a recent mouse study demonstrated that the protective effect of $H$. pylori against allergic diseases did not require live bacteria, treatment with an extract of $H$. pylori in neonates may prevent the development of airway allergic diseases $(48,49)$. The application of $H$. pylori extract, particularly in early life, may be an effective prevention and treatment measure for CVA and AC (43). Of note, the present study had certain limitations. A larger sample size and multicentre prospective clinical trials are required to verify the effectiveness of this novel potential clinical therapeutic strategy.

In conclusion, the results of the present study support the theory of a potential association between a $H$. pylori positive state and $\mathrm{CC}$, including $\mathrm{CRC}, \mathrm{CVA}$ and $\mathrm{AC}$. In addition, HPI may be associated with the accelerated decline of lung function in patients with chronic persistent cough, which may lead to irreversible airway remodelling and even the future development of chronic airway diseases. However, there is an inverse association between HPI and allergic diseases, including CVA and AC. Thus, H. pylori may be a promising target for the treatment of chronic persistent cough. However, further research is required.

\section{Acknowledgements}

Not applicable.

\section{Funding}

This work was supported by grants from Department of Health of Zhejiang Province, China (grant no. 2019KY226) and Shaoxing Bureau of Science and Technology, Zhejiang Province, China (grant no. 2018C30097). It was also supported by Zhejiang Provincial Natural Science Foundation of China under Grant No. LQ20H010001.

\section{Availability of data and materials}

The datasets used and/or analyzed during the current study are available from the corresponding author on reasonable request.

\section{Authors' contributions}

MH and KT designed the research and did most of the work. JS was mainly responsible for the interpretation of data, and also revised it critically for the important intellectual content. $\mathrm{AM}$ and $\mathrm{YZ}$ were mainly responsible for the acquisition and analysis of data. CZ, YY and HW were mainly accountable for ensuring the questions related to the accuracy or integrity of the work to be appropriately investigated and resolved, and performed the experiments. YC and MX also performed some of the experiments. All the authors read and approved the final manuscript.

\section{Ethics approval and consent to participate}

This article does not contain any prospective studies with human participants or animals performed by any of the authors. It was approved by leaders of Shaoxing People's Hospital and registered and approved by the ethics committee of Shaoxing People's Hospital (Shaixing, China). Informed consent was obtained from all individual participants included in the study by telephone interviews.

\section{Patient consent for publication}

Not applicable.

\section{Competing interests}

The authors declare that they have no competing interests.

\section{References}

1. Smith JA and Woodcock A: Chronic cough. N Engl J Med 376: 183-184, 2017.

2. Chung KF: IFN- $\gamma$ : A driver of cough hypersensitivity pathways in chronic cough. Am J Respir Crit Care Med 198: 827-828, 2018.

3. Herregods TVK, Pauwels A, Jafari J, Sifrim D, Bredenoord AJ, Tack J and Smout AJPM: Determinants of Reflux-induced chronic cough. Gut 66: 2057-2062, 2017.

4. Song WJ, Kim HJ, Shim JS, Won HK, Kang SY, Sohn KH, Kim BK, Jo EJ, Kim MH, Kim SH, et al: Diagnostic accuracy of fractional exhaled nitric oxide measurement in predicting cough-variant asthma and eosinophilic bronchitis in adults with chronic cough: A systematic review and meta-analysis. J Allergy Clin Immunol 140: 701-709, 2017.

5. Gibson P, Wang G, McGarvey L, Vertigan AE, Altman KW and Birring SS: Treatment of Unexplained Chronic Cough: CHEST guideline and expert panel report. Chest 149: 27-44, 2016.

6. Millqvist E: The problem of treating unexplained chronic cough. Chest 149: 613-614, 2016.

7. Abdulqawi R, Dockry R, Holt K, Layton G, McCarthy BG, Ford AP and Smith JA: P2X3 receptor antagonist (AF-219) in refractory chronic cough: A randomised, Double-blind, Placebo-controlled phase 2 study. Lancet 385: 1198-1205, 2015.

8. Gopal AK, Schuster SJ, Fowler NH, Trotman J, Hess G, Hou JZ, Yacoub A, Lill M, Martin P, Vitolo U, et al: Ibrutinib as treatment for patients with relapsed/refractory follicular lymphoma: Results from the open-label, multicenter, phase II DAWN study. J Clin Oncol 36: 2405-2412, 2018.

9. Smith JA, Haines J and Yorke J: Taming chronic cough. Thorax 72: 103-104, 2017.

10. Kalhan R, Dransfield MT, Colangelo LA, Cuttica MJ, Jacobs DR Jr, Thyagarajan B, Estepar RSJ, Harmouche R, Onieva JO, Ash SY, et al: Respiratory symptoms in young adults and future lung disease. The CARDIA lung study. Am J Respir Crit Care Med 197: 1616-1624, 2018.

11. Choi IJ, Kim YI and Park B: Helicobacter pylori and prevention of gastric cancer. N Engl J Med 378: 2244-2245, 2018.

12. Abbasi J: Barry marshall and MD: H pylori 35 Years Later. JAMA 317: 1400-1402, 2017.

13. Ehrlich D, Naini B and Mukewar S: A rare cause of gastric wall thickening. Gastroenterology 154: e18-e20, 2018. 
14. Koch KN, Hartung ML, Urban S, Kyburz A, Bahlmann AS, Lind J, Backert S, Taube C and Müller A: Helicobacter urease-induced activation of the TLR2/NLRP3/IL-18 Axis protects against asthma. J Clin Invest 125: 3297-302, 2015.

15. Talaat M, Gad MS, Magdy EA, Aggag SM and Nour YA: Helicobacter pylori infection and chronic, persistent cough: Is there an association. J Laryngol Otol 121: 962-7, 2007.

16. Wang L, Guan Y, Li Y, Liu X, Zhang Y, Wang F, Kong L and Guo Q: Association between chronic respiratory diseases and Helicobacter pylori: A meta-analysis. Arch Bronconeumol 51: 273-278, 2015.

17. Kreuter M, Kirsten D, Bahmer T, Penzel R, Claussen M, Ehlers-Tenenbaum S, Muley T, Palmowski K, Eichinger M, Leider M, et al: Screening for Helicobacter pylori in idiopathic pulmonary fibrosis lung biopsies. Respiration 91: 3-8, 2016.

18. Hong L, Li S, Feng Q, Feng X, Jin Y, Zhao Q and Zhang H: $H$. pylori infection may cause sleep-related laryngospasm for a patient in climacteric. J Pain Symptom Manage 39: e6-e7, 2010.

19. Kefang Lai, et al: Guidelines for diagnosis and treatment of cough (2015). Chinese Journal of Tuberculosis and Respiratory Diseases: 323-354, 2016.

20. Liou JM, Fang YJ, Chen CC, Bair MJ, Chang CY, Lee YC, Chen MJ, Chen CC, Tseng CH, Hsu YC, et al: Concomitant, bismuth quadruple, and 14-day triple therapy in the First-line treatment of Helicobacter pylori: A multicentre, Open-label, randomised trial. Lancet 388: 2355-2365, 2016

21. Chey WD, Leontiadis GI, Howden CW and Moss SF: ACG Clinical Guideline: Treatment of Helicobacter pylori Infection. Am J Gastroenterol 112: 212-239, 2017.

22. Zhao RY, Zhu L, Li L, Chen Q and Yang YJ: The applicability of the 1988 version of the prediction equations for adult normal lung function in Shanghai. Zhonghua Jie He He Hu Xi Za Zhi 34: 586-589, 2011 (In Chinese)

23. Parsonnet J: Clinician-discoverers-Marshall, Warren, and H. pylori. N Engl J Med 353: 2421-2423, 2005.

24. Lanas A and Chan FKL: Peptic ulcer disease. Lancet 390: 613-624, 2017.

25. Kim IJ, Lee J, Oh SJ, Yoon MS, Jang SS, Holland RL, Reno ML, Hamad MN, Maeda T, Chung HJ, et al: Helicobacter pylori Infection modulates host cell metabolism through VacA-dependent inhibition of mTORC1. Cell Host Microbe 23: 583-593.e8, 2018.

26. Kariya S, Okano M and Nishizaki K: An Association between Helicobacter pylori and upper respiratory tract disease: Fact or fiction. World J Gastroenterol 20: 1470-1484, 2014

27. De Korwin JD, Ianiro G, Gibiino G and Gasbarrini A Helicobacter pylori infection and extragastric diseases in 2017. Helicobacter: 22 (Suppl 1), 2017 doi: 10.1111/hel.12411.

28. Gülhan M, Ozyilmaz E, Tarhan G, Demirağ F, Capan N, Ertürk A, Canbakan S, Ayaşlioğlu E, Gülhan E and Ahmed K Helicobacter pylori in bronchiectasis: A polymerase chain reaction assay in bronchoalveolar lavage fluid and bronchiectatic lung tissue. Arch Med Res 38: 317-321, 2007.

29. El-Serag HB, Kao JY, Kanwal F, Gilger M, LoVecchio F, Moss SF, Crowe SE, Elfant A, Haas T, Hapke RJ and Graham DY: Houston consensus conference on testing for Helicobacter pylori Infection in the United states. clin gastroenterol hepatol 16 992-1002.e6, 2018.

30. Rossi AF, Cadamuro AC, Biselli-Perico JM, Leite KR, Severino FE, Reis PP, Cordeiro JA and Silva AE: Interaction between inflammatory mediators and miRNAs in Helicobacter pylori Infection. Cell Microbiol 18: 1444-1458, 2016.

31. Barrozo RM, Hansen LM, Lam AM, Skoog EC, Martin ME, Cai LP, Lin Y, Latoscha A, Suerbaum S, Canfield DR, et al: CagY is an Immune-sensitive regulator of the Helicobacter pylori type iv secretion system. Gastroenterology 151: 1164-1175.e3, 2016.

32. D'Elios MM, Montecucco $\mathrm{C}$ and de Bernard M: VacA and HP-NAP, Ying and Yang of Helicobacter pylori-associated gastric inflammation. Clin Chim Acta 381: 32-38, 2007

33. Ramachandran M, Jin C, Yu D, Eriksson F and Essand M: Vector-encoded Helicobacter pylori neutrophil-activating protein promotes maturation of dendritic cells with Th1 polarization and Improved Migration. J Immunol 193: 2287-2296, 2014.
34. Nakashima S, Kakugawa T, Yura H, Tomonaga M, Harada T, Hara A, Hara S, Nakano M, Yamasaki E, Sakamoto N, et al: Identification of Helicobacter pylori vaca in human lung and its effects on lung cells. Biochem Biophys Res Commun 460: 721-726, 2015.

35. Montecucco $\mathrm{C}$ and de Bernard M: Immunosuppressive and proinflammatory activities of the VacA Toxin of Helicobacter pylori. J Exp Med 198: 1767-1771, 2003.

36. Fullerton D, Britton JR, Lewis SA, Pavord ID, McKeever TM and Fogarty AW: Helicobacter pylori and lung function, asthma, atopy and allergic disease-a population-based cross-sectional study in adults. Int J Epidemiol 38: 419-426, 2009.

37. Gencer M, Ceylan E, Yildiz ZF and Aksoy N: Helicobacter pylori seroprevalence in patients with chronic obstructive pulmonary disease and its relation to pulmonary function tests. Respiration 74: 170-175, 2007.

38. Sze MA, Chen YW, Tam S, Tashkin D, Wise RA, Connett JE, Man SP and Sin DD: The relationship between Helicobacter pylori Seropositivity and COPD. Thorax 70: 923-929,2015.

39. Fogarty AW: Does Helicobacter pylori Infection modify lung development, height or simply reflect shared environmental exposures. Thorax 70: 918, 2015.

40. Wang C, Xu J, Yang L, Xu Y, Zhang X, Bai C, Kang J, Ran P, Shen $\mathrm{H}$, Wen $\mathrm{F}$, et al: China pulmonary health study group. Prevalence and risk factors of chronic obstructive pulmonary disease in China (the China Pulmonary Health [CPH] study): A national Cross-sectional study. Lancet 391: 1706-1717, 2018.

41. Taye B, Enquselassie F, Tsegaye A, Amberbir A, Medhin G, Fogarty A, Robinson K and Davey G: Association between infection with Helicobacter pylori and atopy in young ethiopian children: A longitudinal study. Clin Exp Allergy 47: 1299-1308, 2017.

42. Engler DB, Reuter S, van Wijck Y, Urban S, Kyburz A Maxeiner J, Martin H, Yogev N, Waisman A, Gerhard M, et al: Effective treatment of allergic airway inflammation with Helicobacter pylori immunomodulators requires BATF3-dependent dendritic cells and IL-10. Proc Natl Acad Sci USA 111: 11810-11815, 2014.

43. van Wijck Y, de Kleijn S, John-Schuster G, Mertens TCJ, Hiemstra PS, Müller A, Smits HH and Taube C: Therapeutic application of an extract of Helicobacter pylori ameliorates the development of allergic airway disease. J Immunol 200: 1570-1579, 2018

44. Lim JH, Kim N, Lim SH, Kwon JW, Shin CM, Chang YS, Kim JS, Jung HC and Cho SH: Inverse relationship between Helicobacter pylori infection and asthma among adults younger than 40 years: A cross-sectional study. Medicine (Baltimore) 95: e2609, 2016.

45. van Wijck Y, John-Schuster G, van Schadewijk A, van den Oever RL, Obieglo K, Hiemstra PS, Müller A, Smits HH and Taube C: Extract of Helicobacter pylori ameliorates parameters of airway inflammation and goblet cell hyperplasia following repeated allergen exposure. Int Arch Allergy Immunol 180: 1-9, 2019.

46. Lee SP, Lee SY, Kim JH, Sung IK, Park HS, Shim CS and Moon HW: Correlation between Helicobacter pylori Infection, IgE hypersensitivity, and allergic disease in korean adults. Helicobacter 20: 49-55, 2015.

47. Amberbir A, Medhin G, Abegaz WE, Hanlon C, Robinson K, Fogarty A, Britton J, Venn A and Davey G: Exposure to Helicobacter pylori infection in early childhood and the risk of allergic disease and atopic sensitization: A longitudinal birth cohort study. Clin Exp Allergy 44: 563-571, 2014.

48. Leppold C, Tanimoto T, Ozaki A, Morita T and Sari PV: Helicobacter pylori Vaccination. Lancet 387: 748-749, 2016.

49. Liu H, Liu W, Tan Z, Zeng Z, Yang H, Luo S, Wang L, Xi T and Xing Y: Promoting immune efficacy of the oral Helicobacter pylori Vaccine by HP55/PBCA nanoparticles against the gastrointestinal environment. Mol Pharm 15: 3177-3186, 2018.

This work is licensed under a Creative Commons Attribution-NonCommercial-NoDerivatives 4.0 International (CC BY-NC-ND 4.0) License. 